\title{
Acompanhamento Terapêutico e Análise do Comportamento: Avanços e problemáticas nas definições deste fazer
}

\section{Therapeutic Accompaniment and Behavior Analysis: Progress and issues regarding defining this intervention}

\section{Acompañamiento Terapeutico y Analisis de la Conducta: Progresos y Problematicas en definiciones deste hacer}

\author{
Otávio Beltramello', Nádia Kienen²
}

[1] [2] Universidade Estadual de Londrina I Título abreviado: Análise do Comportamento e Acompanhamento Terapêutico I Endereço para correspondência:
Departamento de Psicologia Geral e Análise do Comportamento, Centro de Ciências Biológicas, Universidade Estadual de Londrina. Rodovia Celso Garcia Cid,
PR 445, Km 380, Campus Universitário. Caixa Postal 10.011 Cep 86.057- 970. Londrina, PR I Email: otaviobeltra@hotmail.com I DOI: 10.18761/PAC.2016.034 


\begin{abstract}
Despite that Therapeutic Accompaniment has been recognized as an important intervention in Psychology and Behavior Analysis, there is no consensus regarding what behavior classes are constituent and could define this intervention. This theoretical-conceptual study aims to critically characterize contributions of Psychology and Behavior Analysis to define this intervention, besides proposing that Behavior Analysis and Teaching Programming have theoretical-conceptual and technological resources to contribute to this definition. Based on the literature review, it was found that Therapeutic Accompaniment has been defined using metaphors and as a subordinate service of a clinical psychologist despite the contributions of Behavior Modification, Behavioral Therapy and Applied Behavior Analysis. It is argued that Behavior Analysis and Teaching Programming provide resources that contribute to a more precise definition of Therapeutic Accompaniment Intervention in Psychology. This might be done by characterizing and systematizing the behavior classes that constitute this intervention through different degrees of complexity.
\end{abstract}

Keywords: Therapeutic Intervention, Psychologist training, Teaching Programming, Behavior classes.

Resumen: Aunque el hacer Acompañamiento Terapeutico tenga su importancia reconocida en la Psicología y en la Análisis de la Conducta, no hay consenso en cuanto a las clases de conductas constituyentes de este hacer y que se ha utilizado para definirlo. Esta reflexion teórica objetivou caracterizar críticamente diferentes contribuciones de la Psicología y de la Análisis de Conducta para la definición de ese hacer, además de proponer que la Análisis de la Conducta y la Programación de Ensiño tienen recursos teórico-conceptuales y tecnológicos para contribuir con esta definición. A partir de las análisis de la literatura, se verificó que el Acompañamiento Terapéutico ha sido definido mediante el uso de metáforas y como un servicio necesariamente subordinado al trabajo del psicólogo clínico, pesar de las contribuciones de la Modificación de la Conducta, Terapia Conductual y Análisis Aplicada de la Conducta. Se argumenta que el Análisis de la Conducta y la Programación de Enseño disponen de recursos para contribuir a una definición más precisa del hacer Acompañamiento Terapéutico en Psicología por medio de la caracterización y sistematización de las clases de conductas que definen este hacer por medio de sus diferentes grados de complejidad.

Palabras-clave: Intervención Terapéutica, formación del Psicólogo, Programación de Enseño, Clases de conducta. 
Uma das explicações para muitas falhas relativas a definir conceitos e resolver problemas na Psicologia parece estar relacionada ao uso que cientistas e psicólogos fazem de metáforas e construtos (Skinner, 1977). Os psicólogos estudam relações entre organismo e ambiente, porém utilizam explicações mentalistas e internalistas para se referir a essas relações e seus constituintes (Skinner, 1977). Tais explicações, por sua vez, são utilizadas como objetivo de estudos psicológicos e têm afastado psicólogos da possibilidade de analisar e produzir resultados a partir de dados sobre as relações entre organismo e ambiente. A problemática aqui está no fato de que, segundo Skinner, não há nenhuma evidência de que o mundo mental se relaciona com as variáveis das quais os comportamentos são função, diferentemente das perspectivas cognitivistas ou psicodinâmicas (como a Psicanálise) que aceitam e estudam esta possibilidade.

Um dos tipos de intervenção realizados por psicólogos que têm atendido pessoas com transtornos psiquiátricos, deficiência física, dependentes químicos, crianças e adolescentes com dificuldades no processo de escolarização e idosos no enfrentamento de situações decorrentes do envelhecimento, é denominado Acompanhamento Terapêutico. Do ponto de vista da Psicologia, de modo geral, essa intervenção pode ser considerada um dos exemplos da problemática apontada por Skinner (1977). Isto por se tratar de um fazer em que comumente se utiliza recursos conceituais explicativos internalistas, com uso frequente de metáforas, na maioria das vezes, vinculados a propostas psicanalíticas. Segundo Nogueira (2009), por exemplo, o Acompanhamento Terapêutico deve ser definido como um fazer que possibilita ao cliente a construção de novas referências para além da equipe multidisciplinar, por meio de um setting não determinado (diversos ambientes e contextos), com o envolvimento de diferentes indivíduos, não necessariamente pertencentes a uma equipe multidisciplinar (o que é denominado "Rede Terapêutica"). Além dessas características, segundo o autor, é um fazer ligado à "identificação" e "manejo de laços com o 'Outro'" (conceito utilizado por Lacan para se referir aos efeitos da incidência da linguagem sobre indivíduos) (Nogueira, 2009). Nesse exemplo, "construção de novas referências" pode ser considerada uma metáfora para designar classes de comportamentos, assim como "envolvimento" se refere a interações comportamentais entre indivíduos e ambientes.

Os dados de uma ciência deveriam guiar os cientistas para a formulação de definições operacionais de termos, por meio de observações, de procedimentos de manipulação e de passos lógicos (que se interpõem entre as observações e os procedimentos de manipulação) (Skinner, 1961). Isso significa que há necessidade científica em desenvolver definições operacionais dos termos, em detrimento do uso da linguagem vernacular (semelhante à utilizada no «senso comum») (Skinner, 1961). No entanto, apesar dos recursos conceituais e tecnológicos produzidos na Análise do Comportamento e, apesar do Acompanhamento Terapêutico ser uma prática comum entre analistas do comportamento, esse fazer também parece ainda não estar claramente definido na própria Análise do Comportamento. Como exemplo, o Acompanhamento Terapêutico tem sido definido por analistas do comportamento como um fazer auxiliar e subordinado ao de um psicólogo clínico e/ou de uma equipe, com execução de procedimentos indicados por esses profissionais, sendo também um «importante elo» entre a família, o cliente e demais pessoas envolvidas (Zamignani, Kovac, \& Vermes, 2007). Essa definição tem como requisito a aceitação de que a "subordinação" do acompanhante terapêutico em uma equipe deve ser uma de suas características definidoras. Esse requisito é um exemplo de uma característica ("subordinação" do acompanhante terapêutico) que não define explicitamente o núcleo do fazer do Acompanhamento Terapêutico, uma vez que indica apenas o tipo de relação a ser estabelecida entre o acompanhante terapêutico e o psicólogo, sem fazer referência aos comportamentos constituintes desse fazer.

A segunda característica ainda presente na definição de Acompanhamento Terapêutico a partir da perspectiva analítico-comportamental e que a afasta do pensamento de Skinner (1977) está na manutenção do uso de metáforas. Expressões tais como importante "elo" (Zamignani, Kovac, \& Vermes, 2007) ou "ferramenta" para "prevenir" que os pacientes se tornem "pacientes críticos" (Cruz, Lima \& Moraes, 2003) têm sido utilizadas para fazer referência a relações entre organismo e ambien- 
te e seus constituintes. Além dos problemas com uso de metáforas, outras definições apresentadas por analistas do comportamento fazem referência ao Acompanhamento Terapêutico como uma forma de intervenção e/ou complemento de um tratamento multidisciplinar (Vianna \& Sampaio, 2003) ou ainda como uma técnica de intervenção (Balvedi, 2003). Esses exemplos ilustram a falta de delimitação, sistematização e consenso em propostas para a definição do Acompanhamento Terapêutico do ponto de vista analítico-comportamental. Sugerem também a falta de estudos na Análise do Comportamento que objetivem a identificação e análise dos comportamentos constituintes desse fazer, o que possibilitaria a proposição de uma definição mais consistente com a perspectiva analítico-comportamental.

O Acompanhamento Terapêutico ocorre por meio de intervenções em ambiente natural do cliente e apresenta algumas particularidades que exigem um repertório clínico bastante sofisticado e um exame acurado das variáveis que o envolvem (Zamignani, Kovac, \& Vermes, 2007). Esse fazer tem acontecido desde a década de 1980 no Brasil e tem trazido benefícios a clientes que não desenvolveram ou que apresentam dificuldades ao emitir comportamentos necessários para desempenharem tarefas da vida cotidiana, e que em geral, para um bom prognóstico, necessitam de vários atendimentos semanais realizados por diferentes especialistas (e.g. psiquiatra, psicólogo e/ou fonoaudiólogo) paralelamente com o serviço de Acompanhamento Terapêutico (Zamignani, 1997; Zamignani \& Wielenska, 1999). Portanto, o Acompanhamento Terapêutico tem sido utilizado para atender diversos casos e em variadas situações, como exemplo, em dificuldades no desempenho escolar, em casos que envolvem drogadição e em situações psiquiátricas graves.

As variáveis que envolvem o repertório clínico necessário para intervenções por meio do Acompanhamento Terapêutico podem ser examinadas de diferentes formas, contando com variados procedimentos e a partir de diferentes teorias e conceitos. Como exemplo, pode-se caracterizá-las por meio da história do Acompanhamento Terapêutico, da descrição dos comportamentos relacionados a esse fazer, ou ainda de acordo com o perfil profissional dos acompanhantes terapêuticos de uma determinada localidade, como o Brasil. $\mathrm{Na}$ Psicologia, apesar das diferentes contribuições teóricas, conceituais e práticas para a definição do Acompanhamento Terapêutico, ainda parece haver a necessidade de um exame mais acurado das variáveis que compõem ou que participam de eventos comportamentais envolvidos nesse fazer. No presente artigo objetivou-se caracterizar de forma crítica as diferentes contribuições da Psicologia e da Análise do Comportamento para a definição de Acompanhamento Terapêutico, problematizando as características que têm sido utilizadas por pesquisadores como definidoras deste fazer no Brasil. Além disso, defende-se que a Análise do Comportamento e a Programação de Ensino possuem recursos para contribuir com uma definição mais precisa do fazer Acompanhamento Terapêutico na Psicologia, por meio de tecnologias de identificação e derivação de comportamentos.

\section{Acompanhamento Terapêutico: origens e produção científica na Psicologia}

A prática que vem sendo denominada por Acompanhamento Terapêutico no Brasil teve suas origens no século XX e em derivações de propostas psicanalíticas. Seus pressupostos surgiram a partir da criação e atribuição de novas funções para agentes de saúde mental, originadas em movimentos político-ideológicos, como o movimento Antipsiquiatria, liderado por Laing e Cooper na Inglaterra, na década de 1960; a Psiquiatria Democrática, representada por Basaglia na Itália, nas décadas de 1970 e 1980; e a Psicoterapia Institucional, caracterizada por Oury e Guattari na França, na década de 1950 (Nogueira, 2009). Esses movimentos contestaram o tratamento psiquiátrico realizado no modelo hospitalocêntrico e possibilitaram o surgimento de novas modalidades de tratamento como as comunidades terapêuticas, o hospital-dia e, posteriormente, o Acompanhamento Terapêutico (Nogueira, 2009; Simões \& Kirschbaum, 2005).

Os serviços originados a partir de contestações ao modelo hospitalocêntrico possibilitaram o surgimento, nos Estados Unidos e na Europa, 
na década de 1950, e na América Latina, na década de 1960, do Acompanhamento Terapêutico (Alvarenga, 2006; Neto, Pinto, \& Oliveira, 2011). O cerne das primeiras funções desse fazer originou-se na Terapia Familiar e na Psicoterapia Institucional. $\mathrm{Na}$ Terapia Familiar, as atribuições do acompanhante terapêutico estavam relacionadas à "diluição da gravidade da doença" entre membros da família e à proposição de alteração de comportamentos do paciente (Ayub, 1996). Já a Psicoterapia Institucional influenciou na formulação da função de "olhar em rede", um procedimento técnico utilizado por alguns acompanhantes terapêuticos para a formulação de projetos terapêuticos realizados em articulações entre uma equipe (Hermann, 2008).

No Brasil, as funções precursoras do Acompanhamento Terapêutico receberam variadas denominações ao longo da história como: Atendente Psiquiátrico, Auxiliar Psiquiátrico, Amigo Qualificado e, finalmente, Acompanhante Terapêutico. A primeira denominação, atendente psiquiátrico, surgiu na Clínica Pinel, em Porto Alegre, nas décadas de 1960 e 1970, sob a filosofia de um Hospital Dinâmico ${ }^{1}$ (Silva $\&$ Silva, 2006). O atendente psiquiátrico era um cargo que não exigia formação acadêmica para ser exercido e cuja função era acompanhar os pacientes internos em suas rotinas (Nogueira, 2009). Já o segundo termo, auxiliar psiquiátrico, surgiu na comunidade terapêutica Clínica Villa Pinheiros, no Rio de Janeiro, em 1969 (Nogueira, 2009) sendo um fazer geralmente exercido por estudantes de psicologia e medicina e que também não exigia formação superior (Nogueira, 2009). Esse serviço continuou a ser requisitado por terapeutas e familiares de pacientes após o fechamento das comunidades terapêuticas no final da década de 1970 (Londero \& Pacheco, 2006; Marco \& Calais, 2012; Nogueira, 2009). Por conta disso, o auxiliar psiquiátrico passou a ser um serviço particular realizado na residência dos pacientes, baseado em pressupostos psicanalíticos e que envolvia principalmente pacientes psicóticos (Londero \& $\mathrm{Pa}$ checo, 2006; Zamignani \& Wielenska, 1999).

1 No Hospital Dinâmico os trabalhos ocorriam por meio de socioterapias, grupos operativos, ambientoterapias, reuniões comunitárias e outras atividades desenvolvidas por equipes de trabalhos, constituídas por psicólogos e assistentes sociais (Silva \& Silva, 2006).
A denominação subsequente, Amigo Qualificado, surgiu em 1981 no instituto A Casa, em São Paulo (Simões \& Kirschbaum, 2005). A função do amigo qualificado era estar junto do paciente em momentos em que este não estava em atividades do Hospital-Dia, principalmente nos finais de semana; e sua denominação foi substituída na mesma década de seu surgimento pela denominação Acompanhante Terapêutico (Nogueira, 2009; Simões \& Kirschbaum, 2005). Essa substituição de denominação ocorreu, segundo os psicanalistas, pela necessidade de desfazer a referência a um vínculo equivocado de simetria entre pacientes e profissionais (Nogueira, 2009). A denominação Acompanhante Terapêutico foi escolhida por ser derivada de termos constituintes da Terapia Familiar e de pressupostos psicanalíticos. "Terapêutico" é um termo que salienta a importância dada à Terapia Familiar para a evolução do tratamento dos pacientes e "Acompanhante" é um termo que retrata uma ação, a de acompanhar, e portanto, segundo os psicanalistas, um bom substituto para o termo (amigo) que descrevia um vínculo equivocado (Nogueira, 2009).

As variações existentes nas denominações dos fazeres relativos ao Acompanhamento Terapêutico resultam do desenvolvimento de conhecimento acerca das variáveis que os constituem. Desde a década de 1980 o Acompanhamento Terapêutico é tema de uma variada produção científica sob diferentes proposições teórico-conceituais (Hermann, 2008; Muylaert, 2006; Pitiá, 2002; Santos, 2013; Zamignani, Kovac, \& Vermes, 2007) e de aplicação na Psicologia no Brasil (Alvarenga, 2006; Marco \& Calais, 2012; Nogueira, 2009; Sereno, 2006; Silva \& Silva, 2006). Um dos fatores para isto está no fato de o Acompanhamento Terapêutico ser um fazer que não possui teoria, procedimentos e normas éticas próprios, embora estas sejam características necessárias para as execuções deste fazer (Nogueira, 2009).

Dada a variedade de produção científica sobre o Acompanhamento Terapêutico na Psicologia e a falta de clareza quanto aos comportamentos constituintes e delimitadores desse fazer, há dificuldade na elaboração de uma definição consensual para a atuação do psicólogo como acompanhante terapêutico. Isso parece ter sinalizado para a necessidade de realização de estudos com o objetivo de caracterizá-lo: (a) historicamente, afirmando que 
o Acompanhamento Terapêutico surgiu de derivações de propostas psicanalíticas e movimentos político-ideológicos, com funções em oposição à internação e que atuou e atua em uma espécie de setting "ambulante" (Alvarenga, 2006; Araújo, 2005; Neto, Pinto, \& Oliveira, 2011; Pitiá \& Santos, 2006); (b) como fazer, atuando em casos que envolvem diversas temáticas, como a inclusão, a drogadição, o alcoolismo, a depressão pós-parto, pacientes com diagnóstico psiquiátrico e pacientes com transtornos orgânicos (Fujihira, 2006; Fraguas \& Berlinck, 2001; Londero \& Pachecho, 2006; Marinho, 2009; Neto \& Amarante, 2013; Palombini, 2006; Sereno, 2006; Silva \& Silva, 2006); e (c) a partir de seu perfil profissional no Brasil, que em geral é um fazer exercido por estudantes, por meio de estágios, onde o acompanhante terapêutico é percebido como coadjuvante no tratamento, mas com papel ativo, de grande importância e que atua "diretamente" no ambiente em que o paciente está inserido (Figueiredo, 2009; Kischbaum \& Rosa, 2003; Marco \& Calais, 2012; Nogueira, 2009).

Nos anos 2000, o Acompanhamento Terapêutico tem sido descrito na literatura como um fazer alternativo à internação, que abarca funções terapêuticas (Alvarenga, 2006; Chaui-Berlinck, 2010; Neto \& Amarante, 2013; Neto, Pinto, \& Oliveira, 2011; Silva \& Silva, 2006; Zamignani, Kovac, \& Vermes, 2007); e funções sociais, como a inclusão (Fraguas \& Berlinck, 2001; Fujihira, 2006; Sereno, 2006) e a garantia de direitos (Chauí-Berlinck, 2010; Muylaert, 2006; Neto \& Amarante, 2013; Neto, Pinto, \& Oliveira, 2011; Palombini, 2006; Silva \& Silva 2006). $\mathrm{Na}$ década de 2010, o primeiro estudo que objetivou realizar uma revisão sistemática sobre a produção científica a respeito do Acompanhamento Terapêutico, na pós-graduação brasileira, foi publicado por Santos et al. (2015). Nesse trabalho, o grupo de autores examinou 43 teses e dissertações concluídas entre os anos de 2000 e 2011 disponíveis no Banco de Teses e Dissertações da Coordenação de Aperfeiçoamento de Pessoal de Nível Superior (CAPES). Os resultados obtidos indicaram que: $62,8 \%$ das referências analisadas foi produzida entre 2007 e 2011; 41,9\% delas classificadas pela CAPES como pertencentes no banco de dados à categoria de «Psicologia» e 25,6\% à categoria de «Tratamento e prevenção psicológica»; a abordagem teórica mais uti- lizada $(48,8 \%)$ foi a Psicanálise; e 79,1\% dos estudos tratavam de relatos de experiência. Embora essa revisão sistemática realizada por Santos et al. tenha sido significativa em indicar de forma quantitativa as produções sobre Acompanhamento Terapêutico na pós-graduação brasileira, o estudo não indicou especificamente o que tem sido produzido por esses estudos, o que envolveria uma análise qualitativa e que poderia indicar demandas específicas de estudos e pesquisas nessa área.

Outro estudo bibliográfico realizado nos anos 2000 foi o de Simões e Kirschbaum (2005), que analisou a produção científica sobre o Acompanhamento Terapêutico no Brasil de 1960 a 2003. Os autores destacaram que nesses estudos, em geral, há uma «preocupação com o estabelecimento de funções e objetivos que determinavam a especificidade do trabalho de Acompanhamento Terapêutico (...)» (p. 395), destacando como características principais do Acompanhamento Terapêutico: o setting ampliado, o diálogo com a família e o trabalho em equipe. Nesse estudo, diferentemente do realizado por Santos et al. (2015), os autores optaram por um procedimento qualitativo, que obteve resultados que parecem corroborar os estudos de Kirschbaum e Rosa (2003) e Londero e Pacheco (2006), demonstrando a demanda de caracterização dos comportamentos constituintes do Acompanhamento Terapêutico.

Em suma, o Acompanhamento Terapêutico tem sido descrito na literatura como um fazer: que não possui teoria, procedimentos e normas éticas próprios (embora essas sejam características necessárias para sua execução); e não privativo de alguma área do conhecimento e profissão. Ambas características contribuem para o aumento de dificuldades na prestação de serviços e na elaboração de uma definição para a atuação de profissionais. Essas dificuldades, por sua vez, parecem ter indicado a necessidade de elaboração de estudos que caracterizassem o Acompanhamento Terapêutico. Essas caracterizações foram realizadas de diferentes maneiras (e.g. historicamente; indicando características deste fazer; ou traçando um perfil profissional dos acompanhantes terapêuticos) e foram importantes por caracterizar esse fazer em três funções principais: (a) sua função terapêutica; (b) sua função social; e (c) sua participação na garantia de direitos (do cliente). 
Uma problemática existente e possivelmente oriunda da variedade teórica, conceitual e de aplicações do Acompanhamento Terapêutico está no fato de que os estudos realizados sobre essa temática, em geral, têm indicado que as características principais do Acompanhamento Terapêutico são: o setting ampliado, o diálogo com a família e o trabalho em equipe. Essas três características parecem se referir às funções do Acompanhante Terapêutico (terapêuticas, sociais e de garantia de direitos) e a situações e contextos nos quais o Acompanhante Terapêutico deve atuar (como no setting ampliado, no diálogo com a família e no trabalho em equipe), mas não explicitam claramente o que esse profissional tem que ser capaz de fazer (seus comportamentos) e que caracterizaria essas funções nesses contextos.

\section{Aproximações entre fazeres da Análise do Comportamento e o Acompanhamento Terapêutico}

A Análise do Comportamento pode contribuir para o desenvolvimento do Acompanhamento Terapêutico por meio de exames de variáveis que compõem ou que participam de eventos comportamentais e que estão envolvidas nesse fazer. Outra contribuição está na aplicação de procedimentos de avaliação e análise de comportamentos realizadas por meio da Modificação do Comportamento, da Análise do Comportamento Aplicada e da Terapia Comportamental. Essas contribuições podem ser exemplificadas a partir do uso de procedimentos de avaliação e de observação de comportamentos "diretamente" no ambiente em que ocorrem e de procedimentos de avaliação e demonstração de relações de determinação entre variáveis comportamentais. $\mathrm{O}$ destaque para a necessidade de que o comportamento-alvo de intervenção tenha relevância social e que as intervenções à que será submetido sejam oriundas de pesquisas básicas, assim como a possibilidade de pesquisas aplicadas, que ocorrem em contexto natural também parecem exemplos de contribuições a serem consideradas.

O Acompanhamento Terapêutico exige procedimentos de observação e de avaliação de comportamentos realizados diretamente no ambiente em que ocorrem (Zamignani, Kovac, \& Vermes, 2007). Esses fazeres se assemelham com os realizados por modificadores do comportamento. A Modificação do Comportamento era composta por procedimentos de observação, avaliação e demonstração de relação de determinação entre variáveis comportamentais. Esses procedimentos ocorriam em conjunto com a transposição ou adaptação de conhecimentos produzidos em pesquisa básica para uso em contextos e ambientes não controlados (Barcellos \& Haydu, 1998; Londero \& Pacheco, 2006; Marco \& Calais, 2012).

A Modificação do Comportamento surgiu diante de um contexto científico em que os estudos eram realizados baseados principalmente no paradigma respondente. Isso perdurou das décadas de 1920 a 1940 (Barcellos \& Haydu, 1998) e teve seu início atribuído ao experimento de Watson e Rayner (1920), os quais demonstraram o condicionamento do "medo", no experimento com o menino Albert. Um dos fatores contextuais para o surgimento da Modificação de Comportamento foram as constantes críticas da Psicologia e da Psiquiatria ao tratamento tradicional em relação ao comportamento tido como "anormal", a partir de abordagens psicodinâmicas como a Psicanálise (Caballo, 1996). Diante disso e do crescente desenvolvimento e influência das ciências biológicas e físicas à Psicologia, teorias da aprendizagem e seus princípios, como os investigados por Pavlov, Thorndike, Watson, Hull, Guthrie e Skinner, ganharam um notável destaque em intervenções. Em grande parte, por seu comprometimento científico evidenciado em suas características: a transposição do modelo de laboratório para a situação clínica, o rigor da produção de conhecimentos para atender a comunidade científica e a prestação de serviços com vistas à promoção de melhoras sociais significativas (Baer, Wolf, \& Risley, 1968).

Ao longo das décadas, pesquisas em diferentes temáticas foram realizadas pela comunidade científica da Análise do Comportamento: como na década de 1930, envolvendo princípios preditivos e proposições teórico-conceituais e na década de 1940, envolvendo o paradigma operante e estudos realizados em infra-humanos, o que pareceu ter conduzido o enfoque principal dos estudos da década posterior, muito marcada pelas contribuições para 
o desenvolvimento da Terapia Comportamental (Barcellos \& Haydu, 1998). O surgimento da Terapia Comportamental, segundo Guedes (1993), é proveniente de críticas direcionadas a modificadores do comportamento, em específico aos procedimentos que adotavam (observação, avaliação e demonstração de relações de determinação entre variáveis comportamentais) e à transposição ou adaptação de conhecimentos produzidos em pesquisa básica para uso em contextos e ambientes não controlados. Segundo as críticas, os procedimentos e a transposição de conhecimentos da pesquisa básica demonstravam que os modificadores do comportamento eram superficiais em suas atuações profissionais e violadores da liberdade pessoal de indivíduos (Guedes, 1993). Considerando as críticas direcionadas aos modificadores do comportamento, parte deles, segundo Guedes (1993), abandonou "(...) a solução de problemas concretos, a rapidez da terapia, os registros, a confiabilidade na relação procedimentos/resultados e a esperança de que a oferta do serviço psicológico, um dia, viesse a ser avaliável pela sociedade" (p. 62). Além disso, deu-se início a novas práticas, como a utilização da análise funcional como ferramenta de análise, a utilização de contingências artificiais, o enfoque no autoconhecimento e a adoção de um setting terapêutico, segundo a autora, não próprio da Análise do Comportamento e que pode ser incoerente com seus princípios.

Outra área que possui características constituintes semelhantes às que compõem o Acompanhamento Terapêutico é a Análise do Comportamento Aplicada, que destacou a necessidade do uso do modelo científico na prestação de serviços, buscando uma integração entre o rigor científico e a intervenção em problemas de relevância social. Conforme Baer, Wolf e Risley (1968), a Análise do Comportamento Aplicada caracteriza-se como uma intervenção em que o comportamento alvo tem sua importância representada por meio de sua relevância social, para o indivíduo ou para a sociedade. Além disso, segundo os autores, trata-se de uma intervenção em que tanto as variáveis envolvidas com o comportamento alvo, quanto a intervenção sobre ele, são claramente controladas, de modo que se possa demonstrar a efetividade dos procedimentos em produzir mudanças so- ciais significativas. Em seus primórdios, a Análise do Comportamento Aplicada partiu da premissa de que o comportamento deveria ser mensurável e observável, o que, como afirma Moskorz, Kubo, De Luca e Botomé (2012), naquela época se referia à resposta do organismo, afastando-se da noção atual de definição do comportamento como “(...) um complexo sistema de contingências (de eventos circunstanciais não necessários, fixos ou com papéis ou funções obrigatórios) que vão configurando, progressivamente, as características das ações ou atividades com as quais cada organismo interage com o "mundo" (determinados aspectos dele) com o qual se defronta" (Botomé, 2001, p.14).

Portanto, no final da década de 1960 e no início da década de 1970, as intervenções contavam com procedimentos que permitiam que variáveis do ambiente fossem manipuladas para promover a alteração de respostas específicas (em geral, alterando sua taxa de ocorrência absoluta e/ou relativa), utilizando-se delineamentos de linha de base múltipla ou procedimentos de reversão (Guedes, 1993; Moskorz, Kubo, De Luca, \& Botomé, 2012). A Análise do Comportamento Aplicada, a Modificação do Comportamento e a Terapia Comportamental parecem ter sido áreas em que a produção científica forneceu importantes contribuições para o Acompanhamento Terapêutico, tais como os procedimentos de observação e intervenção. Isso pode ser inferido e exemplificado a partir da definição apresentada por Zamignani, Kovac e Vermes (2007) para o Acompanhamento Terapêutico a partir de princípios da Análise do Comportamento:

Pode-se resumir o acompanhamento terapêutico como uma intervenção clínica indicada em casos de déficits importantes no repertório básico de comportamentos, o que gera a necessidade de uma atenção intensiva realizada nos locais em que o cliente vive. E o AT é, nesse contexto, o profissional ou estudante "cuja função não compreende analisar o caso e decidir quais atividades e procedimentos utilizar na sua intervenção. Suas ações são, necessariamente, subordinadas às decisões anteriormente elaboradas pelo profissional ou equipe com o/a qual trabalha" (Zamignani \& Wielenska, 1999, 
p. 160) e "que apresenta-se como um elo entre terapeuta, cliente, família e demais pessoas envolvidas, levantando dados importantes para a análise funcional" (Carvalho, 2002, p. 43) (Zamignani, Kovac, \& Vermes, 2007, p. 34-35).

Essa definição abarca características que foram inicialmente utilizadas na então denominada Modificação do Comportamento e na Análise do Comportamento Aplicada, como a utilização de procedimentos de observação, avaliação e demonstração de relações de determinação entre variáveis comportamentais, a necessidade de que o comportamento-alvo de intervenção tenha relevância social e conte com intervenções oriundas de pesquisas básicas e que, na prática, ocorrem em contexto natural. Ainda nessa definição, os autores indicam que o contexto clínico, quando não suficiente para a resolução de uma problemática, leva o clínico a buscar no Acompanhante Terapêutico um auxiliar para a realização do serviço. Portanto, partindo da definição de Zamignani, Kovac e Vermes (2007), pode-se hipotetizar que a definição do Acompanhamento Terapêutico na Análise do Comportamento está relacionada a três fatores principais: (a) o surgimento de procedimentos de intervenção em contexto natural, por meio da Modificação do Comportamento; (b) a delimitação da Análise do Comportamento, por meio da Análise do Comportamento Aplicada e (c) o surgimento da Terapia Comportamental, em intervenções realizadas em setting clínico tradicional, em gabinete.

As contribuições da Análise do Comportamento para o Acompanhamento Terapêutico no Brasil parecem ter surgido em um momento em que houve um aumento no número de profissionais que atuavam em setting clínico em detrimento do número de profissionais que realizavam atendimentos em ambientes e contextos naturais de seus clientes. Por outro lado, as demandas de atendimentos extra-consultório, em casos de desenvolvimento atípico, por exemplo, parecem ter enfatizado a necessidade de "retorno" desses profissionais a intervenções em ambiente natural. Isso porque os profissionais que atuavam exclusivamente na clínica identificaram que, para obter maior eficácia em tratamentos, certos procedimentos teriam que ser aplicados em ou- tros ambientes, como a casa do cliente, e/ou a escola (Zamignani, Kovac, \& Vermes, 2007). Em consonância com isso, pesquisas aplicadas vinham sendo realizadas objetivando desenvolver tecnologia para trabalho em ambiente natural (partindo da premissa de que produziriam maior chance de generalização de resultados obtidos durante a intervenção) (e.g. Asmus et al., 1999; Handleman, 1979; Neef, Iwata, \& Page, 1978). Essas pesquisas contribuíram para o desenvolvimento do Acompanhamento Terapêutico (Cassas, 2013), que parece suprir algumas limitações de profissionais clínicos em atender demandas extra-consultório, como exemplos: a disponibilidade de horário necessária para os atendimentos, os deslocamentos necessários (para ir até o local necessário), a obtenção de dados diretamente observados para realização de análises funcionais e a comunicação mais frequente com agências de controle, como a escola e a família.

Para os analistas do comportamento há uma diferenciação entre o terapeuta analista do comportamento e o acompanhante terapêutico, pois os acompanhantes terapêuticos cumprem a função de auxiliar ou complementar o trabalho de outro terapeuta ou de uma equipe multiprofissional (Balvedi, 2003; Cruz, Lima \& Moraes, 2003; Oliveira, 2000; Vianna \& Sampaio, 2003; Zamignani, Kovac, \& Vermes, 2007; Zamignani \& Wielenska, 1999). As contingências que determinam essa distinção parecem ligadas a aspectos da formação profissional pois, em geral, esse fazer é exercido por estudantes ou terapeutas recém-formados e a questões sociais e econômicas que guiam o Acompanhamento Terapêutico para "uma espécie de estágio remunerado no qual ele acompanha de perto o trabalho de um terapeuta experiente que o supervisiona" (Zamignani, Kovac, \& Vermes, 2007, p. 34). Essa distinção é importante por indicar que o Acompanhamento Terapêutico, na perspectiva de analistas do comportamento, parece um fazer diretamente ligado à formação do psicólogo. Isso, por sua vez, implica em considerar que é preciso criar condições, durante a formação profissional, para que os comportamentos constituintes desse fazer sejam desenvolvidos. Portanto, identificar essas classes de comportamentos se faz importante por possibilitar a caracterização, delimitação e sistematização desse fazer profissional, 
como condição para que o desenvolvimento desses comportamentos ocorra.

As contribuições da Modificação do Comportamento e da Análise do Comportamento Aplicada, em grande parte, influenciam as intervenções realizadas diretamente nas contingências-problema e em diversos contextos diferentes. Portanto, parecem contribuir principalmente para o trabalho do analista do comportamento que atua diretamente nas contingências-problema, por meio de técnicas, procedimentos e utilizando-se de instrumentos para avaliação e verificação de variáveis e comportamentos.

O fazer clínico por vezes impossibilita a saída do terapeuta de sua clínica (por falta de disponibilidade de tempo ou dificuldades de locomoção, por exemplo). Na clínica podem surgir demandas em que há exigência de que o trabalho ocorra em diversos contextos e com um número maior de sessões, e quando isso ocorre, geralmente os terapeutas recorrem ao Acompanhamento Terapêutico (Zamignani, Kovac, \& Vermes, 2007). O acompanhante terapêutico é um fazer relacionado ao manejo de contingências de reforçamento em ambiente natural (Savoia \& Sampaio, 2010). Mesmo que o acompanhante terapêutico seja um psicólogo experiente, como os participantes da pesquisa de Marco (2011), com oito e dezessete anos de experiência em Acompanhamento Terapêutico, ainda é considerado, por definição, como um subordinado ao terapeuta. Isso ocorre por esse fazer ter implícito em sua definição a exclusividade de situações em que é a alternativa financeiramente viável de tratamento, quando é um fazer exercido por estudantes e psicólogos recém-formados (Zamignani, Kovac, \& Vermes, 2007).

Outra problemática existente na Análise do Comportamento para a definição do Acompanhamento Terapêutico está no fato de essa intervenção ainda ser definida apenas parcialmente, sem uma avaliação mais ampla das variáveis que definem esse fazer. Variáveis tais como o controle que o profissional possui sobre o comportamento do cliente em ambiente natural (Cassas, 2013), a posição que o Acompanhamento Terapêutico ocupa em uma equipe (como subordinado) ou a sua função na interação com a família, o cliente e demais envolvidos no processo (Zamignani, Kovac, \& Vermes), o complemento de um tratamento multidisciplinar (Vianna \& Sampaio, 2003) ou ainda uma técnica de intervenção (Balvedi, 2003) parecem contribuir de modo limitado para esclarecer o que é definidor desse fazer. Esses aspectos utilizados como definições indicam parcelas de variáveis constituintes do Acompanhamento Terapêutico, e sinalizam a necessidade de uma avaliação mais minuciosa das classes de comportamentos (e de suas abrangências) que caracterizam esse fazer.

\section{Contribuições da Programação de Ensino ${ }^{2}$ para definir o Acompanhamento Terapêutico por meio de classes de comportamentos}

As funções e objetivos que especificam o fazer de qualquer profissional, inclusive do acompanhante terapêutico, se referem a comportamentos constituintes desse fazer, tais como comportamentos relacionados à intervenção, observação e comunicação. Para os analistas do comportamento, um comportamento representa a interação entre aquilo que o organismo faz e o ambiente no qual realiza esse fazer (Botomé, 2001; 2013). O que o organismo faz representa a sua ação, e o ambiente no qual realiza esse fazer é constituído por dois momentos distintos: o ambiente antecedente, composto por aspectos específicos da situação na qual a ação ocorre e o ambiente subsequente, composto por aspectos específicos que se seguem a ação do organismo (Botomé, 2001; 2013). Esquematicamente é possível representar a noção de comportamento conforme a Figura 1. Nessa tabela são especificados os três componentes do comportamento: a situação que antecede uma ação (ambiente que existe antes do organismo atuar sobre ele), a ação (aquilo que o organismo faz) e a situação que decorre da ação ou sucede a ela (ambiente que resulta dessa ação) (Botomé, 2001; 2013).

2 A denominação mais precisa para a área seria "Análise e Programação de Condições para o Desenvolvimento de Comportamentos“, mas para fins didáticos o nome será resumido como "Programação de Ensino". 
Situação

(Classes de estímulos antecedentes)

O que acontece antes ou junto à ação

do organismo
Ação (Classes de respostas)

Aquilo que o organismo faz
Situação subsequente

(Classes de estímulos consequentes)

O que acontece depois da ação de um organismo

Figura 1 . Especificação dos componentes constituintes da definição do comportamento como interação entre o que o organismo faz e o ambiente em que o faz. As setas representam as intereações entre os componentes do comportamento. (Adaptada de Botomé, 2001)

A noção de comportamento como interação entre atividades do organismo e aspectos do ambiente aumenta a precisão na descrição de comportamentos que são apresentados pelos organismos e viabiliza nomeá-los de forma a indicar a interação estabelecida. Os comportamentos são comumente nomeados fazendo referência à interação que está sendo estabelecida, por meio de um verbo acompanhado de complementos que fazem referência ao ambiente. $\mathrm{O}$ acompanhamento de complementos é necessário, pois a utilização de verbos isolados apenas resume a interação ao invés de indicar os componentes envolvidos na interação entre o que o organismo faz e o ambiente em que o faz (Botomé, 2001; 2013). Como exemplo, uma tentativa de nomear o comportamento "intervir" por meio do uso isolado desse verbo ("intervir") indica apenas a ação de um organismo e não descreve de forma precisa o comportamento do qual essa ação é constituinte: intervir sobre o que? Com quem? Para quê? Como exemplo, o verbo "intervir" pode estar se referindo a um acompanhante terapêutico que necessita "intervir" em uma situação-problema relacionada ao comportamento do cliente com o objetivo de amenizá-la ou diluí-la, ou ainda, pode se referir à intervenção que deve ser realizada junto à família para que ela tenha condições de atuar sobre a situação-problema que envolve o cliente. Esses exemplos se referem a situações diferentes que envolvem contextos e ações distintas, caracterizando-se como comportamentos distintos. No primeiro caso, poder-se-ia pensar num comportamento de "intervir diretamente sobre contingências que mantêm o comportamento-problema do cliente" e, no segundo caso, "intervir por meio de ensino sobre o comportamento de familiares para que eles promovam modificações em contingências que mantêm o comportamento-problema do cliente".

A nomeação de um comportamento somente é possível após análise, que envolve explicitar os prováveis antecedentes de uma classe de respostas, as características dessas classes de respostas e os seus consequentes. Isso significa identificar qual é o comportamento que está ocorrendo e entender as relações entre seus componentes (Botomé, 2001). O termo "classe" aqui se refere a vários eventos com propriedades semelhantes (Catania, 1999). Portanto, podemos nos referir a um conjunto de respostas que são topograficamente parecidas como "classe de respostas" e comportamentos que têm todos a mesma função sobre o ambiente como "classe de comportamentos" (Catania, 1999). Portanto, as classes de comportamentos se referem aos comportamentos que podem variar em relação às propriedades que os constituem, porém mantêm a mesma função (Catania, 1999).

Aqui, cabe destacar que os objetivos de um fazer são sempre relações entre classes de estímulos antecedentes, classes de respostas e classes de estímulos consequentes (Botomé, 2001; 2013). Ou seja, são sempre classes de comportamentos. No Acompanhamento Terapêutico, como exemplo, as classes de estímulos antecedentes são aspectos da realidade com os quais o acompanhante terapêutico se deparará; as classes de respostas designam o que ele deverá ser capaz de fazer em relação a esses aspectos da realidade e as classes de estímulos consequentes se referem ao que necessitará decorrer desse fazer, ou seja, são as mudanças em relação à realidade na qual o acompanhante terapêutico atou. Portanto, quando são propostos objetivos, são propostos comportamentos que necessitarão ser desenvolvidos pelo acompanhante terapêutico para que ele intervenha sobre comportamentos de seus clientes nos contextos em que esses comportamentos ocorrem.

Para uma clara delimitação e sistematização do fazer Acompanhamento Terapêutico, se faz necessário identificar quais as classes de comportamentos dele constituintes, o que implica em compre- 
endê-las, também, a partir de diferentes graus de complexidade. Isso porque as classes de comportamentos constituintes de qualquer fazer profissional variam em complexidade (Kienen, 2008). Como exemplo, tomemos duas classes de comportamento necessárias para um caso de Acompanhamento Terapêutico: "orientar os pais" e "criar condições que gerem sucesso na execução de tarefas escolares" (Pergher \& Velasco, 2007). No exemplo, embora "criar condições que gerem sucesso na execução de tarefas escolares" pareça ser mais complexo do que “orientar os pais", para "orientar" é necessário que o profissional seja capaz de "identificar" e "avaliar" componentes-problemas. "Orientar os pais" pode ser um dos constituintes de "criar condições que gerem sucesso na execução de tarefas escolares", mas requer que sua complexidade seja analisada. Explorar esse aspecto relativo à complexidade das classes de comportamentos é importante por possibilitar identificar comportamentos que necessitam ser desenvolvidos como pré-requisito para o desenvolvimento de outros comportamentos.

Pesquisas que objetivam caracterizar classes de comportamentos constituintes do fazer de diferentes agentes sociais têm sido realizadas por meio de procedimentos de identificação e derivação de comportamentos, especialmente a partir de literatura a respeito do fenômeno investigado. $\mathrm{O}$ estudo de Kienen (2008), por exemplo, buscou delimitar o fazer do psicólogo como um profissional capacitador de diferentes agentes sociais (pais, cônjuges, profissionais da área da saúde, líderes empresariais etc.) para que eles possam aprender a intervir sobre comportamentos. Santos, Kienen, Viecili, Botomé e Kubo (2009) examinaram as classes de comportamentos constituintes da formação do psicólogo a partir do que está proposto nas Diretrizes Curriculares para os Cursos de Graduação em Psicologia. Moskorz (2011) investigou os comportamentos presentes na interação entre psicoterapeuta e cliente, aprofundando o exame feito por Zamignani (2007). Luiz (2008) sistematizou as classes de comportamentos constituintes de "projetar a vida profissional". De Luca (2008) caracterizou a classe "avaliar a confiabilidade de informações". Esses são exemplos de estudos que objetivaram caracterizar e sistematizar classes de comportamentos constituintes de algum fazer, a partir da literatura, o que possilitou avaliar a pertinência dessas classes, realizar proposições com a finalidade de amenizar equívocos presentes na linguagem utilizada para se referir a elas, bem como em problemáticas decorrentes desses equívocos, além de caracterizar funções sociais e científicas de profissionais e permitir a capacitação de indivíduos que necessitam lidar com comportamentos envolvidos nessas funções.

No caso do Acompanhamento Terapêutico, a utilização de procedimentos de identificação e derivação de comportamentos a partir de literatura sobre esse tipo de fazer, pode auxiliar na elaboração de uma definição e em sua delimitação. A seguir será apresentado um exemplo para ilustrar o potencial desse tipo de procedimento, iniciando com a transcrição de um trecho do texto de Oliveira (2000) que apresenta uma definição para o Acompanhamento Terapêutico:

"O AT serve de elo entre o profissional ou a equipe responsável e a família. Dessa forma, o AT pode reforçar adequadamente os esforços tanto do cliente quanto de sua família, orientar o cliente em suas tarefas diárias, garantido o exercício das atividades programadas; realizar o levantamento de dados da relação familiar e de contingências da vida do paciente, através de observação participante; repassar os dados novos, levantados através da observação ao profissional ou equipe responsável pelo caso, para a análise das novas contingências percebidas e assim traçar novos procedimentos e técnicas cabíveis ao caso" (Oliveira, 2000, p. 257).

Nesse trecho, é possível identificar referência aos três componentes que constituem comportamentos: classes de estímulos antecedentes, classes de respostas e classes de estímulos consequentes. Como classes de estímulos antecedentes, pode-se destacar: Cliente, esforços do cliente, esforços da família, atividades programadas, profissional ou equipe responsável pelo caso, tarefas diárias do cliente. Como classes de respostas: Reforçar adequadamente os esforços tanto do cliente quanto de sua família; orientar o cliente em suas tarefas diárias garantindo o exercício das atividades programadas; realizar o levantamento de dados da relação familiar e de contingências da vida do pacien- 
te, através de observação participante; e repassar os dados novos, levantados através da observação ao profissional ou equipe responsável pelo caso. Por fim, como referências às classes de estímulos consequentes, é possível observar: a garantia do exercício das atividades programadas, e duas decorrências do comportamento do acompanhante terapêutico que são classes de comportamentos que deverão ser emitidos pelo profissional ou equipe responsável pelo caso: análise de novas contingências percebidas e traçar novos procedimentos e técnicas cabíveis ao caso. Nesses dois últimos casos, os estímulos consequentes de classes de respostas do acompanhante terapêutico poderiam ser mais claramente descritas como: possiblidade de análise de novas contingências percebidas, possibilidade de que novos procedimentos sejam traçados, possibilidade de novas técnicas cabíveis ao caso sejam traçadas. Porém, mesmo explicitando aspectos relativos aos três componentes de comportamentos, as expressões utilizadas para fazer referência aos comportamentos do acompanhante terapêutico parecem não deixar clara a sua função. Isso porque são mantidas metáforas ("serve de elo", "através", "percebidas", entre outras) e uma ênfase às classes de respostas que o acompanhante terapêutico deve emitir ("reforçar", "orientar", "realizar", "através de observação participante", "repassar os dados novos"). Uma maneira de explicitar mais claramente as classes de comportamentos destacadas nesse trecho seria substituir os termos vagos, metafóricos ou imprecisos por termos mais claros, concisos, objetivos e precisos ${ }^{3}$, levando em consideração a função dessas classes de comportamentos. Como

3 Objetividade se refere à linguagem utilizada para descrever os componentes que fazem referência a variáveis observáveis (direta ou indiretamente) (De Luca, 2008, 2013);

A concisão é um critério que define que a linguagem não deve apresentar palavras ou expressões desnecessárias (De Luca, 2008, 2013);

Clareza define que a linguagem utilizada deve ser de fácil compreensão, indicando variáveis constituintes de comportamentos (de seus componentes e/ou de uma classe de comportamentos) (De Luca, 2008, 2013);

Precisão é um critério relacionado às medidas das variáveis de comportamentos (de seus componentes e/ou de classes de comportamentos), fazendo referência à amplitude dos valores das variáveis (quanto menor a amplitude, mais precisa a informação é) (De Luca, 2008, 2013). exemplo, a classe "realizar o levantamento de dados da relação familiar e de contingências da vida do paciente, através de observação participante" poderia ser substituída por "coletar dados de interações comportamentais entre membros da família do cliente por meio de observações participantes". Essa expressão parece destacar mais claramente o tipo de ação a ser executada pelo acompanhante terapêutico. Por sua vez, essa classe de respostas poderia, ainda, ser decomposta em outras classes menos abrangentes, tais como "coletar dados sobre contingências de reforçamento que envolvem o paciente", "observar interações comportamentais entre membros da família do cliente", assim como classes relativas a avaliar, caracterizar e identificar esses mesmos dados e essas mesmas interações.

Vale destacar ainda que, além de propor a substituição de alguns termos, é possível, a partir do trecho destacado, propor a derivação de outros componentes de classes de comportamentos que podem ser inferidos a partir das informações apresentadas. Como exemplo, é possível destacar consequências tais como: Dados comunicados para membros da equipe ou ao responsável pelo caso; dados sobre interações comportamentais da família do cliente obtidos; exercício das atividades programadas garantido; interações comportamentais entre membros da família do cliente identificadas, caracterizadas, avaliadas, observadas, entre outras.

Como ilustrado aqui, procedimentos de identificação e derivação de classes de comportamentos a partir da literatura sobre o fenômeno investigado possibilitam aumentar a clareza a respeito dos comportamentos definidores da atuação do acompanhante terapêutico. Esse procedimento, além da identificação de componentes de comportamentos, possibilita a operacionalização dos termos, aumentando a clareza a respeito dos comportamentos definidores desse fazer profissional. Ao realizarmos um procedimento de identificação e derivação de componentes de comportamentos (seus componentes e classes de comportamentos) no trecho de Oliveira (2000), podemos identificar referências a diversas classes de comportamentos relativas a identificar, caracterizar, avaliar, observar e intervir (sobre interações comportamentais, por exemplo). Após identificação, derivação e avaliação das expressões apresentadas no trecho, é possível propor, 
inclusive, uma nova versão de redação desse, com uma terminologia mais consistente. Neste caso, o trecho poderia ser substituído por: "O acompanhante terapêutico serve como mediador de relações comportamentais entre o profissional ou a equipe responsável pelo caso e a família do cliente. De seus comportamentos decorrem a possibilidade de análise de contingências e de desenvolvimento de procedimentos e técnicas que serão utilizadas nesse caso. Portanto, trata-se de um fazer responsável por identificar, caracterizar, avaliar, observar, coletar e comunicar (para a equipe ou profissional responsável pelo caso) dados sobre contingências de reforçamento que envolvem o cliente e sobre interações comportamentais entre membros de sua família. Também são funções do Acompanhante Terapêutico aumentar a probabilidade do cliente e de sua família executarem adequadamente as atividades programadas, orientar o cliente em suas atividades diárias e analisar, avaliar, caracterizar e identificar: relações comportamentais entre o profissional (ou a equipe responsável pelo caso) e a família do cliente; as atividades programas e a probabilidade do cliente e de sua família executarem essas atividades.".

Os exemplos apresentados não têm a pretensão de esgotar o exame do trecho transcrito, nem tampouco definir, por si só, a atuação do acompanhante terapêutico. Trata-se tão somente de ilustrar a possibilidade de uso de procedimentos derivados dos trabalhos que têm sido realizados com Programação de Ensino como forma de definir mais claramente as atuações requeridas do acompanhante terapêutico.

\section{Considerações finais}

$\mathrm{Na}$ Psicologia, como na Análise do Comportamento, há falta de consenso quanto à definição do fazer denominado Acompanhamento Terapêutico, de suas características e suas funções. Isso contribui para a manutenção de lacunas na prestação de serviços, como a dificuldade em realizar verificações de eficácia de procedimentos adotados por acompanhantes terapêuticos e a ausência de documentos norteadores compostos por diretrizes para a atuação profissional do psicólogo por meio do acompanha- mento terapêutico. Tanto a dificuldade em realizar verificações de eficácia de procedimentos adotados, quanto a ausência de documentos norteadores (referentes ao trabalho do psicólogo como acompanhante terapêutico), são problemáticas relacionadas às características utilizadas como definidoras do Acompanhamento Terapêutico.

A definição de Acompanhamento Terapêutico utilizada por analistas do comportamento pressupõe que esse fazer se define pela intervenção sobre contingências de reforçamento em ambiente natural. Porém, o acompanhante terapêutico tem sido considerado um profissional subordinado ao psicólogo clínico e sem uma especificação clara dos comportamentos constituintes de seu fazer. Essa definição a partir da subordinação restringe o trabalho de psicólogos, à medida que não considera o trabalho de psicólogos acompanhantes terapêuticos que são parte de uma equipe onde não há subordinação de um psicólogo a outro, ou em equipes onde o acompanhante terapêutico é o único psicólogo. Ademais, há o uso de metáforas ou de componentes de comportamentos (e/ou componentes de classes de comportamentos) ao invés de classes de comportamentos constituintes e definidoras deste fazer.

A Análise do Comportamento como ciência, e a Programação de Ensino como uma subárea desta ciência, possuem recursos teórico-conceituais e tecnológicos para contribuir com uma melhor definição do fazer Acompanhamento Terapêutico na Psicologia. Como exemplos, há a possibilidade de identificar as classes de comportamentos constituintes do Acompanhamento Terapêutico, bem como identificar seus diferentes graus de abrangência, tal como realizado em estudos que objetivaram descobrir classes de comportamentos componentes de fazeres, profissões e funções sociais (Botomé, 1997; De Luca, 2008; Kienen, 2008; Luiz, 2008; Moskorz, 2011; Santos et al., 2009). Nesses estudos, os autores utilizaram a tecnologia de identificação e derivação de comportamentos, a partir de aspectos constituintes desses comportamentos, que possibilitou que os comportamentos fossem submetidos à avaliação experimental e/ou à construção de relações entre esses aspectos (sínteses comportamentais), por meio de procedimentos de ensino (Kienen, Kubo, \& Botomé, 2013). 
Identificar e derivar componentes de comportamentos (classes de estímulos antecedentes e consequentes e classes de respostas) e/ou comportamentos a partir da literatura, com base nesses recursos teórico-conceituais e tecnológicos provenientes da Análise do Comportamento e da Programação de Ensino parece um meio promissor para delimitar o fazer denominado Acompanhamento Terapêutico. Além disso, possibilita também maior visibilidade sobre o que necessita ser ensinado para os futuros profissionais que pretendem atuar como acompanhantes terapêuticos.

\section{Referências:}

Alvarenga, C. (2006). Trânsitos da clínica do acompanhamento terapêutico (AT): Da via histórica à cotidiana (Dissertação de mestrado). Universidade Federal de Uberlândia, Uberlândia. Disponível em: http://www.pgpsi.ip.ufu.br/sites/pgpsi.ip.ufu.br/files/Anexos/ Bookpage/DissertacaoCeriseAlvarenga.pdf

Araújo, F. (2005). Do amigo qualificado à política da amizade. Estilos da clínica. 10(19), 84-105. Disponível em: http://pepsic.bvsalud.org/scielo. phpscript=sci_arttext\&pid=S141571282005000 200005\&lng=pt\&tlng=pt.

Asmus, J.M., Wacker, D.P., Harding, J., Berg, W.K., Derby, K.M., \& Kocis, E. (1999). Evaluation of antecedent stimulus parameters for the treatment of escape-maintained aberrant behavior. Journal of Applied Behavior Analysis, 32, 405513. DOI: 10.1901/jaba.1999.32-495

Ayub, P. (1996). Do amigo qualificado ao acompanhante terapêutico. Infanto: Revista de Neuropsiquiatria da Infância e Adolescência, 4(2), 37-40.

Baer, D. M., Wolf, M. M., \& Risley, T. R. (1968). Some current dimensions of applied behavior analysis. Journal of Applied Behavior Analysis, 1(1), 91-97. DOI: 10.1901/jaba.1968.1-91

Balvedi, C. (2003). Acompanhamento terapêutico: A terapia no ambiente do paciente. In M. Z. S. Brandão et al (Orgs), Sobre comportamento e cognição: A história e os avanços, a seleção por consequências em ação (Vol. 11, pp. 294-299). Esetec: Santo André.
Barcellos, A. B. \& Haydu, V. B. (1998). História da psicoterapia comportamental. In B. Rangé (Org.), Psicoterapia comportamental e cognitiva: Pesquisa, prática, aplicações e problemas. (pp. 16-34). Campinas: Editorial Psy.

Botomé, S. P. (1997). Um procedimento para encontrar os comportamentos que constituem as aprendizagens envolvidas em um objetivo de ensino. Não publicado.

Botomé, S. P. (2001) Sobre a noção de comportamento. In H. P. M. Feltes \& U. Ziles (Orgs.), Filosofia: Diálogo de horizontes (pp. 685-708) Porto Alegre: EDIPUCRS.

Botomé, S. P. (2013). O conceito de comportamento operante como problema. Revista Brasileira de Análise do Comportamento, 9(1), 19-46. DOI: http://dx.doi.org/10.18542/rebac.v9i1.2130

Caballo, V. E. (1996). Manual de técnicas de terapia e modificação do comportamento. (M. D. Claudino Trad.) São Paulo: Livraria Santos. Cassas, F. A. (2013). O Acompanhamento Terapêutico como prática do analista do comportamento: uma caracterização histórica com base no behaviorismo radical. (Tese de Doutorado). Pontifícia Universidade Católica de São Paulo. Disponível em: https://sapientia.pucsp.br/handle/handle/16716

Catania, A. C. (1999). Aprendizagem: Comportamento, linguagem e cognição (D. G. De Souza et al. Trads.). Porto Alegre: Artmed (trabalho original publicado em 1998).

Chauí-Berlinck, L. (2010). O acompanhamento terapêutico e a formação do psicólogo: Por uma saúde humana. Arquivos Brasileiros de Psicologia, 62(1), 90-96.

Cruz, F. C.; Lima, L. A. De \& Moras, P. B. de (2003). Acompanhamento terapêutico \& clínica escola: Um novo campo de exploração. In M. Z. S. Brandão et al (Org.), Sobre comportamento e cognição: A história e os avanços, a seleção por consequências em ação (Vol. 11, pp. 300-308). Santo André: ESETec

De Luca, G. G. (2008). Características de componentes de comportamentos básicos constituintes da classe geral de comportamentos denominada "avaliar a confiabilidade de informações" (Dissertação de mestrado). Universidade Federal de Santa Catarina, Florianópolis. 
Disponível em: https://repositorio.ufsc.br/ xmlui/handle/123456789/91008

De Luca, G. G. (2013). Avaliação da eficácia de um programa de contingências para desenvolver comportamentos constituintes da classe geral "avaliar confiabilidade de informações" (Tese de doutorado). Universidade Federal de Santa Catarina, Florianópolis. Disponível em: https://repositorio.ufsc.br/xmlui/handle/123456789/122722

Figueiredo, C. D. (2009). Intervenções realizadas por acompanhantes terapêuticos e a promoção de autonomia na pessoa acompanhada (Monografia de graduação). Universidade do Sul de Santa Catarina, Palhoça.

Fraguas, V., \& Berlinck, M. T. (2001). Entre o pedagógico e o terapêutico: Algumas questões sobre o acompanhamento terapêutico dentro da escola. Dossiê estilos da clínica, 7-16. DOI: http://dx.doi.org/10.11606/issn.1981-1624. v6i11p7-16

Fujihira, C. Y. (2006). Acompanhando a inclusão: Um percurso ético. Psychê, 18(10), 101108. Disponível em: http://pepsic.bvsalud.org/ scielo.php?script $=$ sci_arttext\&pid $=S 1415$ $-11382006000200010 \& \operatorname{lng}=p t \& t \operatorname{lng}=p t$

Guedes, M. L. (1993). Equívocos da terapia comportamental. Temas em Psicologia, 1(2), 8185. Disponível em: http://pepsic.bvsalud.org/ scielo.php?script $=$ sci_arttext\&pid $=S 1413$ $-389 X 1993000200011 \& \operatorname{lng}=\mathrm{pt}$

Handleman, J.S. (1979). Generalization by autistic-type Children of verbal responses across settings. Journal of Applied Behavior Analysis, 12, 273-282. DOI: 10.1901/jaba.1979.12-273

Hermann, M. C. (2008). Acompanhamento terapêutico e psicose: Um articulador do real, simbólico e imaginário (Tese de doutorado). Universidade de São Paulo, São Paulo. DOI: 10.11606/T.47.2008.tde-01122009-105523

Kienen, N. (2008). Classes de comportamentos profissionais do psicólogo para intervir, por meio de ensino, sobre fenômenos e processos psicológicos, derivadas a partir das diretrizes curriculares, da formação desse profissional e de um procedimento de decomposição de comportamentos complexos (Tese de doutorado). Universidade Federal de Santa Catarina, Florianópolis.
Disponível em: https://repositorio.ufsc.br/handle/123456789/92016

Kirschbaum, D. I. R. \& Rosa T. M. (2003). Os trabalhadores de enfermagem como acompanhantes terapêuticos de um centro de atenção especial. Revista Escola Enfermagem da USP, 37 (1), 97-106. Disponível em: http://www.scielo. br/scielo.php?script $=$ sci_arttext\&pid $=$ S0080$-62342003000100012 \& \operatorname{lng}=$ en. http://dx.doi. org/10.1590/S0080-62342003000100012

Londero, I. \& Pacheco, J. T. B. (2006). Por que encaminhar ao acompanhante terapêutico? Uma discussão considerando a perspectiva de psicólogos e psiquiatras. Psicologia em Estudo, 11(2), 259-267. DOI: http://dx.doi.org/10.1590/ S1413-73722006000200004

Luiz, E. C. (2008). Classes de comportamentos componentes da classe geral "projetar a vida profissional", organizadas em um sistema comportamental (Dissertação de mestrado). Universidade Federal de Santa Catarina, Florianópolis. Disponível em: https://repositorio.ufsc.br/handle/123456789/90921

Marco, M. N. (2011). Acompanhante terapêutico: Caracterização da prática profissional na perspectiva da Análise do Comportamento (Dissertação de mestrado). Universidade Estadual Paulista "Júlio de Mesquita Filho", Bauru. Disponível em: http://www2.fc.unesp.br/ BibliotecaVirtual/DetalhaDocumentoAction. do?idDocumento $=364$

Marco, M. N. da C., \& Calais, S. L. (2012). Acompanhante terapêutico: Caracterização da prática profissional na perspectiva da análise do comportamento. Revista Brasileira de Terapia Comportamental e Cognitiva, 14(3), 4-18. Disponível em: http://www.usp.br/rbtcc/ index.php/RBTCC/article/view/546/364

Marinho, D. M. (2009). Acompanhamento terapêutico: Caminhos clínicos, políticos e sociais para a consolidação da reforma psiquiátrica brasileira (Dissertação de mestrado). Universidade de São Paulo, São Paulo. DOI: 10.11606/D.7.2009. tde-14082009-092412

Moskorz, L. (2011). Classes de comportamentos profissionais constituintes da classe geral de comportamentos do psicoterapeuta derivadas de um sistema de categorização de comportamen- 
tos desse tipo de profissional na interação com cliente (Dissertação de mestrado). Universidade Federal de Santa Catarina, Florianópolis. Disponível em: https://repositorio.ufsc.br/handle/123456789/95801

Moskorz, L.; Kubo, O. M.; De Luca, G. G. De, \& Botomé, S. P. (2012). Um exame dos fundamentos para diferentes denominações das intervenções do analista do comportamento em contexto clínico. Acta Comportamentalia, 20(3). 343-365. Disponível em: http://www.revistas. unam.mx/index.php/acom/article/view/35004

Muylaert, M. A. (2006). AT como dispositivo clínico: Uma perspectiva da esquizoanálise, Psychê, 18 (10), 109-114. Disponível em: http:// pepsic.bvsalud.org/scielo.php?script $=$ sci_ arttext\&pid=S1415-11382006000200011\&lng= $\mathrm{pt} \& \mathrm{t} \operatorname{lng}=\mathrm{pt}$

Neef, N.A., Iwata, B.A., \& Page, T. (1978). Public Transportation Training: in vivo versus classroom instruction. Journal of Applied Behavior Analysis, 11(3), 331-344. DOI: 10.1901/ jaba.1978.11-331

Neto, M. de L. A., \& Amarante, P. D. de C. (2013). O acompanhamento terapêutico como estratégia de cuidado na atenção psicossocial. Psicologia: Ciência e profissão, 33(4), 964-975. Disponível em: http://www.redalyc.org/articulo.oa?id=282029760014

Neto, R. de O. R., Pinto, A. C. T., \& Oliveira, L. G. A. (2011). Acompanhamento terapêutico: História, clínica e saber. Psicologia: Ciência e profissão, 31(1), 30-39. DOI: http://dx.doi. org/10.1590/S1414-98932011000100004

Nogueira, A. B. (2009). O acompanhamento terapêutico e sua caracterização em Betim e Belo Horizonte. Psicologia em Revista, 15(2), 214 222. DOI: http://dx.doi.org/10.1590/S141498932011000100004

Oliveira, S. G. (2000). O acompanhante terapêutico. In R. R. Kerbauy (Org.). Sobre comportamento e cognição - Psicologia comportamental e cognitiva: Conceitos, pesquisa e aplicação, a ênfase no ensinar, na emoção e no questionamento clínico (Vol. 5, pp. 257-260). São Paulo, SP: ARBytes.

Palombini, A. de L. (2006). Acompanhamento terapêutico: Dispositivo clínico-político. Psychê, 18(10), 115-127. Disponível em: http:// pepsic.bvsalud.org/scielo.php?script $=$ sci arttext\&pid=S1415-11382006000200012\&lng= pt\&tlng $=p t$

Pergher, N. K. \& Velasco, S. M. (2007). Modalidade de acompanhamento terapêutico para desenvolvimento de comportamentos pró-estudo. IN D.R. Zamignani, R. Kovac, \& J.S. Vermes. A clínica de portas abertas: Experiências e fundamentação do acompanhante terapêutico e da prática clínica clínica em ambiente extraconsultório. (pp. 285-306). Santo André: ESETec.

Pitiá, A. C. de A. (2002). Acompanhamento Terapêutico sob o enfoque da psicoterapia corporal: uma clínica em construção (Tese de doutorado). Universidade de São Paulo, Ribeirão Preto. DOI: 10.11606/T.83.2002.tde-30102006150004

Pitiá, A. C. de A., \& Santos, M. A. dos. (2006). O acompanhamento terapêutico como estratégia de continência do sofrimento psíquico. Revista eletrônica Saúde Mental Álcool e Drogas, 2(2). Disponível em: http://pepsic.bvsalud.org/ scielo.php?script $=$ sci_arttext $\&$ pid $=\mathrm{S} 1806$ $-69762006000200008 \& \operatorname{lng}=\mathrm{pt} \& t \operatorname{lng}=\mathrm{pt}$

Santos, R. G. (2013). Acompanhamento Terapêutico de pacientes neurológicos: Uma experiência de ensino em psicanálise (Tese de doutorado). Universidade de São Paulo, São Paulo. DOI: 10.11606/T.47.2013.tde-08102013-144925

Santos, M. A. dos, Mishima-Gomes, F. K. T., Pillon, S. C., Zanetti, A. C. G., Souza, J. de, Miasso, A. I., \& Peres, R. S. (2015). Produção científica sobre Acompanhamento Terapêutico (AT) na pós-graduação brasileira: Revisão da literatura. Psicologia: teoria e prática, 17(2), 64-77. Disponível em: http://pepsic.bvsalud. org/scielo.php?script=sci_arttext\&pid=S1516$-36872015000200005 \& \operatorname{lng}=p t \& t \operatorname{lng}=\mathrm{pt}$

Santos, G.C.V. dos, Kienen, N., Viecili, J., Botomé, S.P., \& Kubo, O.M. (2009). Habilidades e competências a desenvolver na capacitação de psicólogos: Uma contribuição da Análise do Comportamento para o exame das Diretrizes Curriculares. Interação em Psicologia, 13(1), 131-145. DOI: http://dx.doi.org/10.5380/psi. v13i1.12279

Savoia, M. G., \& Sampaio, T. P. A. (2010). Técnicas cognitivo-comportamentais: Considerações so- 
bre o repertório do AT. IN I. Londero (Org.) Acompanhamento terapêutico: Teoria e técnica na terapia comportamental e cognitivo - comportamental (pp. 37-49). São Paulo: Editora Santos.

Sereno, D. (2006). Acompanhamento terapêutico e educação inclusiva. Psychê, 18(10), 167179. Disponível em: http://pepsic.bvsalud. org/scielo.php?script=sci_arttext\&pid=S1415$-11382006000200016 \& \operatorname{lng}=p t \& t \operatorname{lng}=p t$

Silva, A. S. T. da., \& Silva, R. N. da. (2006). A emergência do acompanhamento terapêutico e as políticas de saúde mental. Psicologia: Ciência e profissão, 26(2), 210-221. DOI: http://dx.doi. org/10.1590/S1414-98932006000200005

Simões, C. H. D., \& Kirschbaum, D. I. R. (2005). Produção científica sobre o acompanhamento terapêutico no Brasil de 1960 a 2003: Uma análise crítica. Revista Gaúcha de Enfermagem, 26(3), 392-402. Disponível em: http://seer.ufrgs. br/index.php/RevistaGauchadeEnfermagem/ article/view/4569/2496

Skinner, B. (1977). Why I am not a cognitive psychologist. Behaviorism, 5(2), 1-10. Disponível em: http://www.jstor.org/ stable/27758892?origin=JSTOR-pdf

Skinner, B. F. (1961). The operational analysis of psychological terms. In Cumulative Record. (pp. 272-286). Appleton: New York. (Publicação original: Psychological Review (1945), 52, 270277).

Vianna, A. M., \& Sampaio, T. P. de A. (2003). Acompanhamento terapêutico: Da teoria à prática. In M. Z. S. Brandão et al. (org). Sobre comportamento e cognição: A história e os avanços, a seleção por consequências em ação (Vol. 11, pp. 285-193). Santo André: ESETec

Zamignani, D. R. (1997). O trabalho de acompanhamento terapêutico: A prática de um analista do comportamento. Revista Biociências, 3(1), 77-90.

Zamignani, D. R. (2007). O desenvolvimento de um sistema multidimensional para a categorização de comportamentos na interação terapêutica (Tese de doutorado). Universidade de São Paulo, São Paulo. DOI: 10.11606/T.47.2008.tde21052009-091808
Zamignani, D. R.; Kovac, R., \& Vermes, J. S. (2007). A clínica de portas abertas: Experiências e fundamentação do acompanhante terapêutico e da prática clínica em ambiente extraconsultório. São Paulo: ESETec.

Zamignani, D. R., \& Wielenska, R. C. (1999). Redefinindo o papel do acompanhante terapêutico. In R.R. Kerbauy, \& R.C. Wielenska. (Orgs.), Sobre comportamento e cognição: Psicologia comportamental e cognitiva da reflexão teórica à diversidade na aplicação (Vol. 4, pp. 157-165). Santo André: ARBytes.

Watson, J. B., \& Rayner, R. (1920) Conditioned emotional reactions. Journal of Experimental Psychology, 3(1), 1-14. DOI: http://dx.doi. org/10.1037/0003-066X.55.3.313

\section{Informações do Artigo}

Histórico do artigo:

Submetido em: 13/09/2016

Primeira decisão editorial: 28/11/2016

Aceito em: 13/03/2017

Editor: Nicodemos B. Borges 